\title{
Calibrating a high-resolution wavefront corrector with a static focal-plane camera
}

\author{
Visa Korkiakoski, ${ }^{1}$ Niek Doelman, ${ }^{2,1}$ Johanan Codona, ${ }^{3}$ \\ Matthew Kenworthy, ${ }^{1}$ Gilles Otten, ${ }^{1}$ and Christoph U. Keller ${ }^{1}$ \\ ${ }^{1}$ Leiden Observatory, Leiden University, P.O. Box 9513, 2300 RA Leiden, The Netherland\$ \\ ${ }^{2}$ TNO Technical Sciences, Stieltjesweg 1, 2628 CK Delft, The Netherlands \\ ${ }^{3}$ Steward Observatory, University of Arizona, Tucson, AZ 85721, USA
}

compiled: November 21, 2021

\begin{abstract}
We present a method to calibrate a high-resolution wavefront-correcting device with a single, static camera, located in the focal-plane; no moving of any component is needed. The method is based on a localized diversity and differential optical transfer functions (dOTF) to compute both the phase and amplitude in the pupil plane located upstream of the last imaging optics. An experiment with a spatial light modulator shows that the calibration is sufficient to robustly operate a focal-plane wavefront sensing algorithm controlling a wavefront corrector with $\sim 40000$ degrees of freedom. We estimate that the locations of identical wavefront corrector elements are determined with a spatial resolution of $0.3 \%$ compared to the pupil diameter.
\end{abstract}

OCIS codes: $\quad 110.1080,110.4850,100.5070,070.6120,120.5050$

\section{Introduction}

In certain situations, such as exoplanet imaging, it is necessary to have an extremely good wavefront (WF) quality. To achieve this, the wavefront must be corrected with a very high resolution; the next-generation extremely large telescopes having apertures larger than $20 \mathrm{~m}$ require deformable mirrors with up to $200 \times 200$ actuators. Furthermore, additional WF sensing must be done at the focal plane to avoid the slowly evolving, non-common path aberration errors.

A possible WF reconstruction algorithm for this purpose is the Fast \& Furious (F\&F) algorithm [1, 2]. It is numerically extremely efficient, relying on small WF aberrations, pupil symmetries and phasediversity to achieve very fast WF reconstruction.

To validate the $\mathrm{F} \& \mathrm{~F}$ algorithm for high-order wavefront correction, we use an inexpensive spatial light modulator (SLM), based on twisted-nematic liquid crystals. It has about $300 \times 300$ wavefrontmodifying pixels surrounding the aperture. The device is able to make a stroke of $\sim 1 \mathrm{rad}$ while maintaining a sufficiently uniform transmittance.

\footnotetext{
* korkiakoski@strw.leidenuniv.nl
}

A successful operation of the system requires precise knowledge of how the SLM reacts to the control signal. In addition, it is necessary to know, with sub-pixel accuracy, where the SLM elements are located with respect to the physical pupil. Information about the pupil amplitudes is important for high-contrast applications, particularly when an SLM causes amplitude aberrations.

Therefore, it is highly desirable to have a method that detects both the phase and amplitude changes in the pupil plane without the need to physically move the imaging camera or wavefront correcting elements.

We found that a single method, simple and easy to implement, is sufficient for all our calibration purposes: the differential optical transfer function method (dOTF) [3-5].

In this paper, we show results from optical experiments demonstrating how the dOTF method can be used for high-contrast imaging calibrations. In Section 2 we describe the theoretical background for the calibration method. Section 3 discusses practical issues in the experiments, and Section 4 shows the results of the dOTF algorithm and the highresolution F\&F performance. Finally, Section 5 draws the conclusions. 


\section{Theoretical background}

The dOTF method is a phase-diversity technique that reconstructs the electric field at the pupil-plane using intensity measurements at the focal plane. However, the method is unique in not requiring any models or a priori information: nothing has to be known about the pupil function, wavefront corrector or the PSF sampling. On the contrary, the dOTF method can be used to calculate, with good accuracy, the parameters required by more conventional phase-diversity algorithms, discussed, for instance, by [6, 7].

The key is a very localized diversity at the edge of the pupil. Once the diversity is close to a delta function, it becomes possible to directly extract the pupil-plane complex amplitudes from the intensity measurements. However, this approach has a major challenge: the image with a delta-function diversity is almost identical to the original image.

The comparison of two very similar images results in issues with the signal-to-noise ratio (SNR). To counteract the SNR problem, we found, as discussed in Section 3.A, that it is extremely helpful to apply a large defocus when applying the dOTF method. Therefore, the following discussion is geared towards applying the dOTF method for calibration purposes, where only the change of the pupil-plane complex amplitudes is of interest and not the image itself.

We begin by describing the basic principles of the dOTF method in Section 2.A. Section 2.B discusses the dOTF accuracy in our setup, and Section 2.C explains how to determine the PSF sampling with the dOTF method.

\section{A. Basic principle of dOTF method}

When imaging a monochromatic point source, the image can be modeled using Fraunhofer diffraction. The image in the focal plane is the squared modulus of the complex amplitudes in the pupil plane,

$$
p=\mathscr{F}\{A \exp (i \phi)\}[\mathscr{F}\{A \exp (i \phi)\}]^{*},
$$

where $\phi$ is the wavefront in the pupil, $A$ is the absolute value of the electric field in the aperture (also describing the pupil shape), and * denotes the complex conjugate. An example of such an image is shown in Fig. 1.

The optical transfer function is the Fourier transform of Eq. (1) and can be written as

$$
P=A \exp (i \phi) * A^{\prime} \exp \left(-i \phi^{\prime}\right)
$$

where $A^{\prime}$ and $\phi^{\prime}$ denote the mirrored versions of $A$ and $\phi: A^{\prime}(x, y)=A(-x,-y), \phi^{\prime}(x, y)=\phi(-x,-y)$.

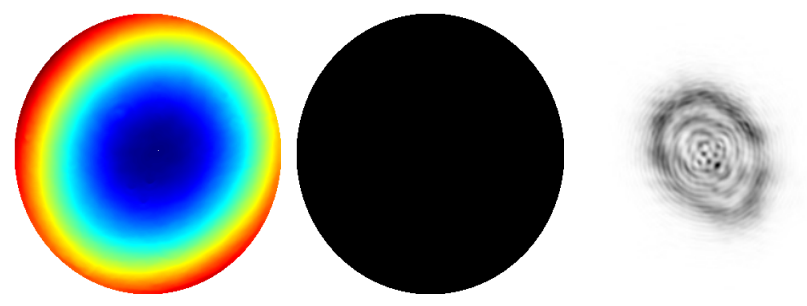

Fig. 1. A simulated example of a PSF. Left: wavefront having a large defocus, 30 rad peak-to-valley, on top of a high-order component with peak-to-valley $\sim 2 \mathrm{rad}$. The wavefront is obtained from laboratory measurements (see Section 4 .A), scaled to a size of $334 \times 334$ pixels. Middle: perfect circular pupil having the same size. Right: the resulting image (shown area is $220 \times 220$ pixels, the total simulated size is $1146 \times 1146$ pixels).

It is convenient to consider the OTF as a convolution of complex functions,

$$
P=\Psi * \Psi^{\prime},
$$

where $\Psi$ denotes the complex amplitudes at the pupil plane, and $\Psi^{\prime}$ is its mirrored and conjugated variant.

To solve the complex amplitudes using only intensity measurements, it is necessary to introduce a diversity - a modification in the wavefront and/or pupil transmittance - and to record another image. The OTF of the diversity image can be written as

$$
P_{2}=\left(\Psi+\Psi_{d}\right) *\left(\Psi^{\prime}+\Psi_{d}^{\prime}\right),
$$

where $\Psi_{d}$ is the change in complex amplitudes in the pupil.

The difference of the the optical transfer functions is

$$
P_{d}=P_{2}-P=\Psi * \Psi_{d}^{\prime}+\Psi^{\prime} * \Psi_{d}+\Psi_{d} * \Psi_{d}^{\prime} .
$$

If the diversity is highly localized, then $\Psi_{d}$ is approximately a delta function (multiplied by a complex constant). A simulated example of such a difference is shown in Fig. 2, The wavefront and pupil are the same as in Fig. 1. The pupil width is 334 pixels, and the diversity is a disk, 15 pixels wide, at the edge of the pupil.

Highly localized means that the range of the diversity is less than $\sim 1-5 \%$ of the pupil width; in such cases it has nonzero values only close to itself. If the diversity is at the edge of the pupil, the two first terms in Eq. (5) have nonzero values at different locations - except for close to the diversity. This can be further illustrated by inspecting the different terms in Eq. (5) separately, as is done in Fig. 3 . 


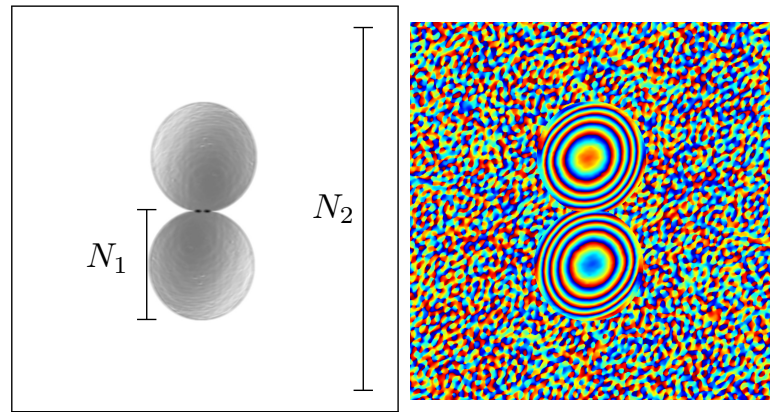

Fig. 2. A simulated dOTF $\left(P_{d}\right)$. The array size is $1146 \times$ 1146 pixels. Left: modulus $-N_{1}$ indicates the pupil diameter in pixels, and $N_{2}$ is the size of the whole dOTF array. Right: phase.

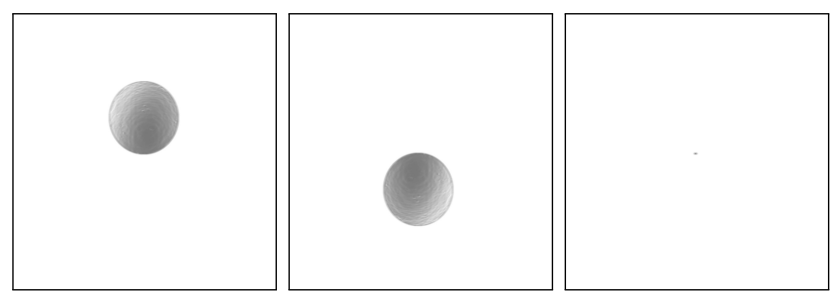

Fig. 3. Moduli of the simulated terms in Eq. (5): $\Psi * \Psi_{d}^{\prime}$, $\Psi^{\prime} * \Psi_{d}$ and $\Psi_{d} * \Psi_{d}^{\prime}$.

The two pupil functions are shifted because the peaks of the convolving diversity functions, $\Psi_{d}$ and $\Psi_{d}^{\prime}$, are separated by about one pupil diameter. The pupil amplitudes are somewhat distorted, since $\Psi_{d}$ is not a perfect delta function. The impact of the third term, $\Psi_{d} * \Psi_{d}^{\prime}$, is negligible in most cases.

Thus, it is possible to consider Eq. (5) piecewise,

$$
\begin{aligned}
& P_{d}(x, y)=\Psi * \Psi_{d}^{\prime} \approx \Psi * \delta_{d}^{\prime}, \quad(x, y) \in D \\
& P_{d}(x, y)=\Psi^{\prime} * \Psi_{d} \approx \Psi^{\prime} * \delta_{d}, \quad(x, y) \in D^{\prime},
\end{aligned}
$$

where $D$ and $D^{\prime}$ denote the regions on opposite sides of the diversity location, and $\delta_{d}$ and $\delta_{d}^{\prime}$ are delta functions multiplied by a complex constant.

\section{B. Analysis of dOTF based calibration}

The measurement error of the dOTF method is almost entirely caused by the smoothing convolution with $\Psi_{d}$ and noise propagation. For our purposes, it is also necessary to have a more detailed look at the error made when characterizing the performance of the SLM. We used the SLM itself to generate the localized diversity, but a similar effect could also be achieved by using an additional device [5].

A numerical example of the convolution bias in a typical measurement situation was set up with a small, constant, WF difference of $0.5 \mathrm{rad}$ and a transmittance reduction of $10 \%$ introduced in one half of the pupil; the undistorted case is the same as shown in Fig. 1. Two dOTF measurements (in total four images) are recorded: both the undistorted and the modified complex amplitudes are determined. These two dOTF arrays are then used to determine the change in the pupil plane. We measure the phase change (denoted as $\Delta_{\phi}$ ) as a subtraction of reconstructed wavefronts. The change in transmittance $\left(\Delta_{A}\right)$ is measured as a ratio of dOTF moduli. It holds that

$$
\begin{aligned}
\Delta_{\phi} & =\text { phase }\left(\Psi_{2} * \Psi_{d 2}^{\prime}\right)-\operatorname{phase}\left(\Psi * \Psi_{d}^{\prime}\right) \\
\Delta_{A} & =\frac{\left|\Psi_{2} * \Psi_{d 2}^{\prime}\right|}{\left|\Psi * \Psi_{d}^{\prime}\right|},
\end{aligned}
$$

where phase $(\cdot)$ denotes a function unwrapping the phase of a complex number, $\Psi_{2}$ is the true complex amplitude after diversity modification, and $\Psi_{d 2}^{\prime}$ describes the localized diversity of the dOTF measurement made for the modified case. In our tests, we always had high SNR, and therefore we did not observe issues with $\left|\Psi * \Psi_{d}^{\prime}\right|$ being zero.

The arrays $\Delta_{\phi}$ and $\Delta_{A}$ are shown in Fig. 4
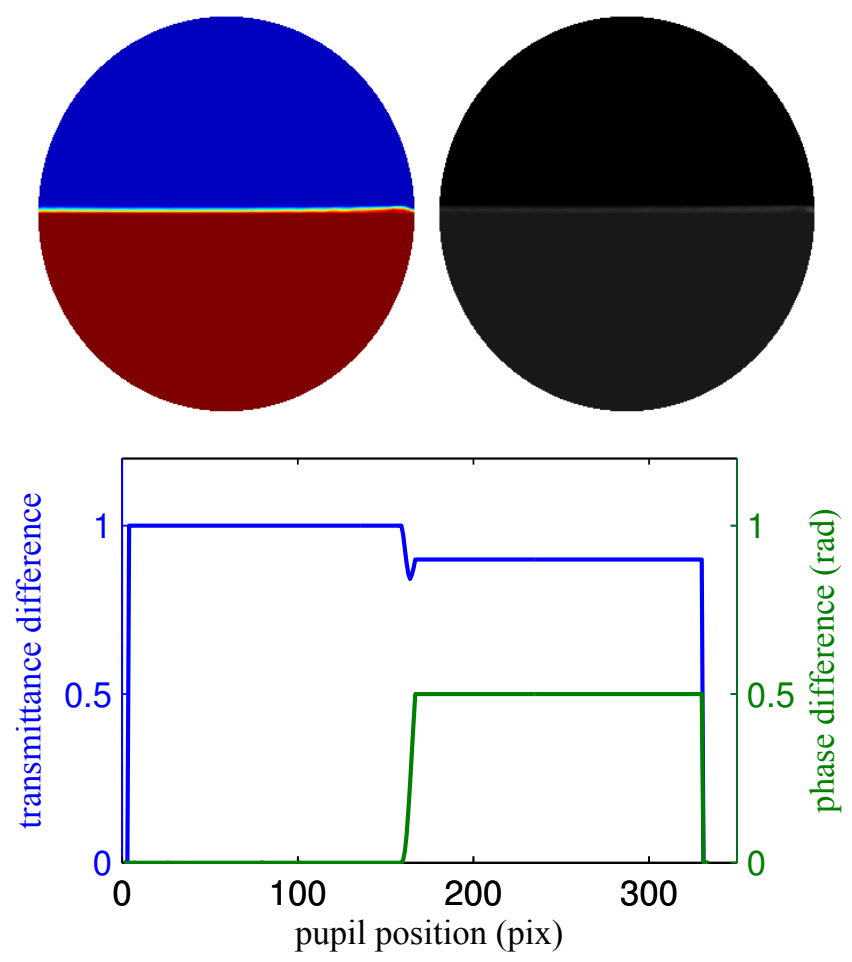

Fig. 4. Illustration of the change in the pupil plane, measured by the dOTF method. Top left: $\Delta_{\phi}$. Top right: $\Delta_{A}$. Bottom: vertical cuts of $\Delta_{\phi}$ and $\Delta_{A}$.

Both the phase and transmittance changes are observed without any bias. Only the convolution smoothing distorts the determined complex amplitudes - it is especially visible along the edge of the modification. The edge appears as a linear transition in the phase, but in the transmittance, the 
edge also has an undershoot of $\sim 5 \%$.

It is particularly interesting that the transmittance change, $\Delta_{A}$, shows no signs of the dOTF modulus distortions, visible for instance in Figs. 2 and 3 . This obviously holds only when the introduced phase and transmittance changes are small enough, but this was the case with all the measurements presented in this paper - the introduced phase change never exceeded $1.6 \mathrm{rad}$.

The numerical example shown is not an extensive analysis of the dOTF method; a more thorough approach would be needed to characterize its limits, which is outside the scope of this paper.

\section{C. Sampling}

Another important issue is the sampling at the detector array. Typically, the sampling is expressed in terms of the width of the diffraction-limited PSF, in pixels, and its value is determined both by the optical design and the detector properties.

Determining the sampling accurately is important for all model-dependent focal plane wavefront sensing techniques. When the PSF is modeled numerically with an FFT, its diffraction-limited width is usually defined as

$$
w=\frac{N_{\text {arr }}}{N_{\text {pup }}},
$$

where $N_{\text {arr }}$ is the width of the zero-padded FFT array in pixels, and $N_{\text {pup }}$ is the diameter of the modeled pupil in pixels. It can be difficult to determine values for $w$ that accurately match the modeled system, if only distorted PSF images are available.

However, the dOTF method offers a straightforward way to determine $w$ with an accuracy close to the dOTF array discretization limit; a greater precision would require dealing with the fixed sampling of the FFT [8]. First, the dOTF array is obtained by Fourier transforming pre-processed and subtracted detector intensities. Then, the value of $w$ is obtained directly: it is the ratio between the total width of the dOTF array and the observed pupil diameter in pixels - indicated by $N_{2}$ and $N_{1}$ in Fig. 2.

Obviously, a successful use of the dOTF method requires that the detector array is at least Nyquist sampled (two pixels per diffraction-limited PSF core) - a limitation when compared to general phase-retrieval methods based on error metric minimization [9]. If the sampling was smaller $(w<2)$, aliasing would occur. Its concrete manifestation would be that the joined pupils (as shown in Fig. 2) would not fit into the FFT array, and this would render the dOTF method useless. To avoid this with a safe margin, we used a moderate oversampling, $w \approx 3$. A larger over-sampling would work as well, but it would require more detector pixels and bigger arrays to handle the data.

\section{Experimentals considerations}

We explain the experimental arrangements we used to test the dOTF method by describing our highdynamic range (HDR) imaging approach (Section 3.A followed by a description of the optical setup (Section 3.B).

\section{A. Defocused high dynamic range imaging}

In principle, the dOTF method could be implemented by taking two consecutive images that have a very localized diversity near the pupil border. In practice, we found that obtaining a high SNR is the biggest challenge. This is not surprising when considering Eq. (5) where two almost identical images are subtracted. Furthermore, those images are the focal plane images of a point-source, which means that - in the absense of large wavefront aberrations - most of the light is localized in the core of a small PSF.

A large defocus during the dOTF measurements spreads the light more evenly over the detector pixels. This improves the SNR and dramatically increases the accuracy of the higher spatial frequencies of the complex amplitudes.

In addition, we paid attention to other aspects that improve the performance. Our image acquisition recipe for a single $\mathrm{dOTF}$ measurement is:

- Create a defocus of 17-30 rad peak-to-valley. Make sure the system is as stable as possible during the recording process. For instance, avoid using deformable mirrors with high voltages to reduce the effects of amplifier noise.

- Adjust the laser power (or change neutral density filters) such that the camera can work with short exposure times (on the order of milliseconds). This reduces the effect of turbulence inside the optical setup and slowly drifting SLM characteristics.

- Select optimal diversity. We used a disk having a width of $2.5-4 \%$ of the pupil diameter (corresponding to 5-8 SLM pixels). Place the center of the diversity at the detected pupil border (half of the disk inside the aperture, as illustrated in Fig. 5). A small disk makes the SNR low, a wide disk blurs and biases the recorded complex amplitudes. We used 
the SLM to introduce the maximum possible complex amplitude change within the diversity disk: $1.6 \mathrm{rad}$ phase difference and $25 \%$ drop in transmittance - a value also found in [5].

- Record a set of images with varying exposure times such that long-exposure images saturate. Create compiled images (as discussed later) to further increase the camera's dynamic range and reduce its read-out noise. This is also called high dynamic range imaging (HDR) [10. We used 5-6 different exposure times $(0.24-29 \mathrm{~ms})$ for one compiled image.

- Create a set of image compilations and average to further reduce the noise. We did this pairwise: first one reference image compilation, then one diversity image compilation, then again one reference and so on. This avoids issues with internal turbulence (caused by air convection) and slow drifts of the SLM. We typically used 40 such pairs for a single dOTF determination.

Our total recording time for one dOTF reconstruction was $2-4 \mathrm{~min}$.

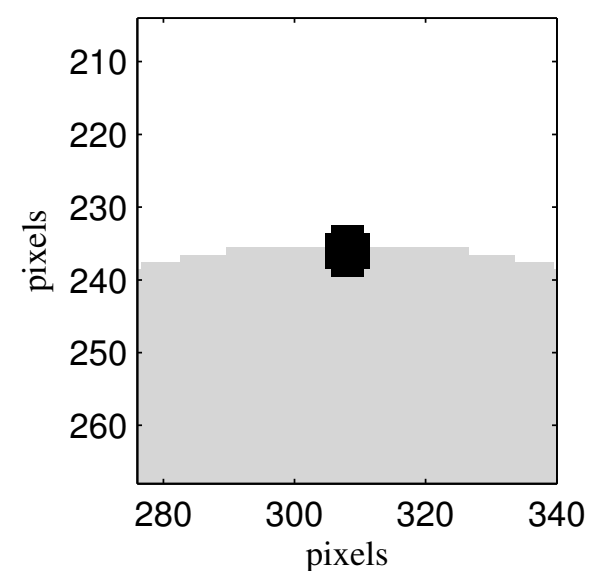

Fig. 5. Illustration of the SLM mask we used to create the diversity for the dOTF method. Black circle shows the modified diversity pixels, gray pixels show the estimated pupil border.

To create the compilation with increased dynamic range, we take a set of $N$ images $\left(I_{1}, I_{2}, \ldots, I_{N}\right)$, ordered according to increasing exposure time. We start by setting $I=I_{1}$. For the remaining images $(k=2, \ldots, N)$, we iteratively update the compilation according to:
1. Calculate a scaling factor

$$
s_{k}=\frac{\sum_{\Omega_{1}} I}{\sum_{\Omega_{1}} I_{k}},
$$

where $\Omega_{1}$ are the unsaturated pixels with adequate signal in both the current compilation $(I)$ and $I_{k}$.

2. Update the compilation: $I\left\{\Omega_{2}\right\}=s_{k} I_{k}\left\{\Omega_{2}\right\}$, where $\Omega_{2}$ is the set of pixels that are not saturated in $I_{k}$.

The linear range and the saturation of the camera are determined by inspecting the response when gradually increasing the exposure time. To be on the safe side, we assume the linear range to be 153500 analog-to-digital units (ADUs) while the maximum intensity of the camera is 4096 ADUs.

\section{B. Optical setup}

A schematic view of the optical setup is shown in Fig. 6. The beam is directed through a linear polarizer, the spatial light modulator and another linear polarizer. Then, it is reflected by a deformable mirror (DM) and focused onto the camera.

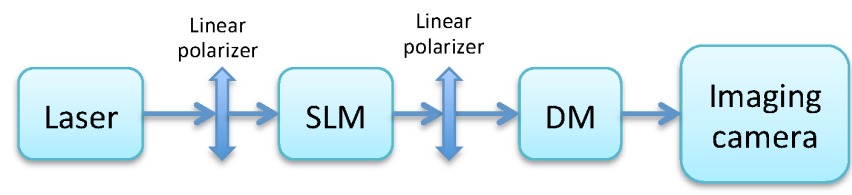

Fig. 6. Schematic view of our optical setup.

The rotation of the polarizers with respect to the SLM is chosen such that the SLM produces maximum phase shifts and minimum transmittance changes (as explained for instance in [11]).

The light source is a laser diode having a wavelength of $656 \mathrm{~nm}$. The laser is coupled to a fiber, and the light is roughly $50 \%$ polarized when arriving at the first collimating lens. We found that the quality of the laser was not a limiting factor, and we made no attempts to study its stability or install a spatial filter in front of the laser window.

Using standard 1 inch doublet lenses, the beam is collimated, passes a diaphragm $\sim 5 \mathrm{~mm}$ wide and is re-collimated to pass the SLM and DM with a diameter of $\sim 1 \mathrm{~cm}$.

The SLM is a transmissive device having $800 \times 600$ pixels. The birefringence of each SLM pixel can be controlled by a VGA signal having a range of 8 bits per pixel (grayscale values between 0-255). For the experiments reported in this paper, we adjusted the 
size of the diaphragm such that the beam passes an area of 334 pixels wide.

The SLM causes a strong diffraction effect since the pixels and wiring between them act as a grating. Several sub-beams emerge, but only the brightest hits the camera. We observe no adverse effects from the other beams.

In addition to the SLM, we use the DM to control the low-order modes of the wavefront. During the dOTF measurements, the DM is not needed, and we turn it off. However, when testing algorithms to achieve a flat wavefront (see Section 4.D), we use the DM to overcome the stroke limitations of the SLM.

The whole setup was built, for convenience, on a $30 \times 45 \mathrm{~cm}$ breadboard with two folding mirrors (before the 1st polarizer and before the imaging camera). The detailed parameters are listed in Table 1.

Table 1. Details of used hardware

\begin{tabular}{|c|c|}
\hline \multicolumn{2}{|r|}{ Imaging camera } \\
\hline Model & Basler piA640-210gm \\
\hline Resolution & $648 \times 488$ pixels \\
\hline Dynamic range & 12 bits \\
\hline Pixel size & $7.4 \times 7.4 \mu \mathrm{m}$ \\
\hline Readout noise & 14 electrons \\
\hline Sensor type & CCD \\
\hline \multicolumn{2}{|c|}{ Spatial light modulator } \\
\hline Model & Holoeye LC2002 \\
\hline Resolution & $800 \times 600$ pixels \\
\hline Fill-factor & $55 \%$ \\
\hline Dynamic range & 8 bits \\
\hline Pixel pitch & $32 \mu \mathrm{m}$ \\
\hline \multicolumn{2}{|c|}{ Deformable mirror } \\
\hline Manufacturer & Flexible Optical B.V. \\
\hline Type & Micromachined membrane DM \\
\hline Diameter & $15 \mathrm{~mm}$ \\
\hline Number of channels & 37 \\
\hline Controlled modes & 36 \\
\hline \multicolumn{2}{|r|}{ Light source } \\
\hline Model & Qphotonics QFLD-660-2S \\
\hline Type & Laser diode, fiber coupled \\
\hline Central wavelength & $656 \mathrm{~nm}$ \\
\hline Spectral bandwidth & $0.7 \mathrm{~nm}$ \\
\hline
\end{tabular}

\section{Results}

This section describes the results of our laboratory experiments. Section 4.A illustrates the properties of the basic dOTF method. Sections $4 . \mathrm{B}$ and $4 . \mathrm{C}$ show the details of how we determined the SLM response and registration with respect to the pupil. Finally, Section 4.D describes the experimental verification of a focal-plane wavefront sensing algorithm.

\section{A. Basic features of dOTF arrays}

First, we show a basic example of the dOTF method when the DM is turned off and all the SLM pixels are set to zero. We also optimized the camera focus and neutral density filters such that short exposure times $(0.24 \mathrm{~ms})$ were possible with a large defocus ( $\sim 30$ rad peak-to-valley) without saturating the camera.

We followed the procedure described in Section 3.A with 5 different exposure times (0.24$4 \mathrm{~ms}$ ). In this way, the dynamic range of the images was $1 \cdot 10^{7}$ to $5 \cdot 10^{7}$ (ratio of smallest and largest non-zero values). For convenience, we cropped the central region of $320 \times 320$ pixels from these HDR images; that region contains enough information for the dOTF reconstruction. Then, we zero-pad the cropped parts to obtain arrays of $640 \times 640$ pixels to avoid FFT wrap-around effects.

The dOTF arrays were then computed as discussed in Section 2.A. We applied no windowing function to avoid blurring the fine features, in particular the pupil borders. An example of the resulting arrays is shown in Fig. 7 .

The dOTF modulus very clearly shows the same pattern as seen in the simulated example in Section 2.A. Due to measurement errors, additional noise is seen. Particularly outside of the circular pupil, the noise can easily be characterized: it consists of small clots, $4-7$ pixels wide. The noise rms is $\sim 3.5 \%$ of the maximum intensity, and its peaks are always lower than $\sim 10 \%$ of the maximum. Ideally, the noise should be almost white since the PSF measurements are limited by the read-out noise. However, the zero-padding, the SLM drift and the internal turbulence cause more complicated errors.

The separation of the two pupils is very clear, which indicates that the applied diversity was close to a delta function. The very localized diversity makes it possible to see fine-scale structures: the shape of the used diaphragm, location and impacts of several dust particles and larger, ring-like structures, whose origin is not clear at this time.

In comparison to the simulated dOTF with a perfect pupil, as discussed in section 2.B, the border effect (a bright ring with lower intensity inside the pupil) is an expected diffraction effect. The measurement bias is also very similar to what is seen in 

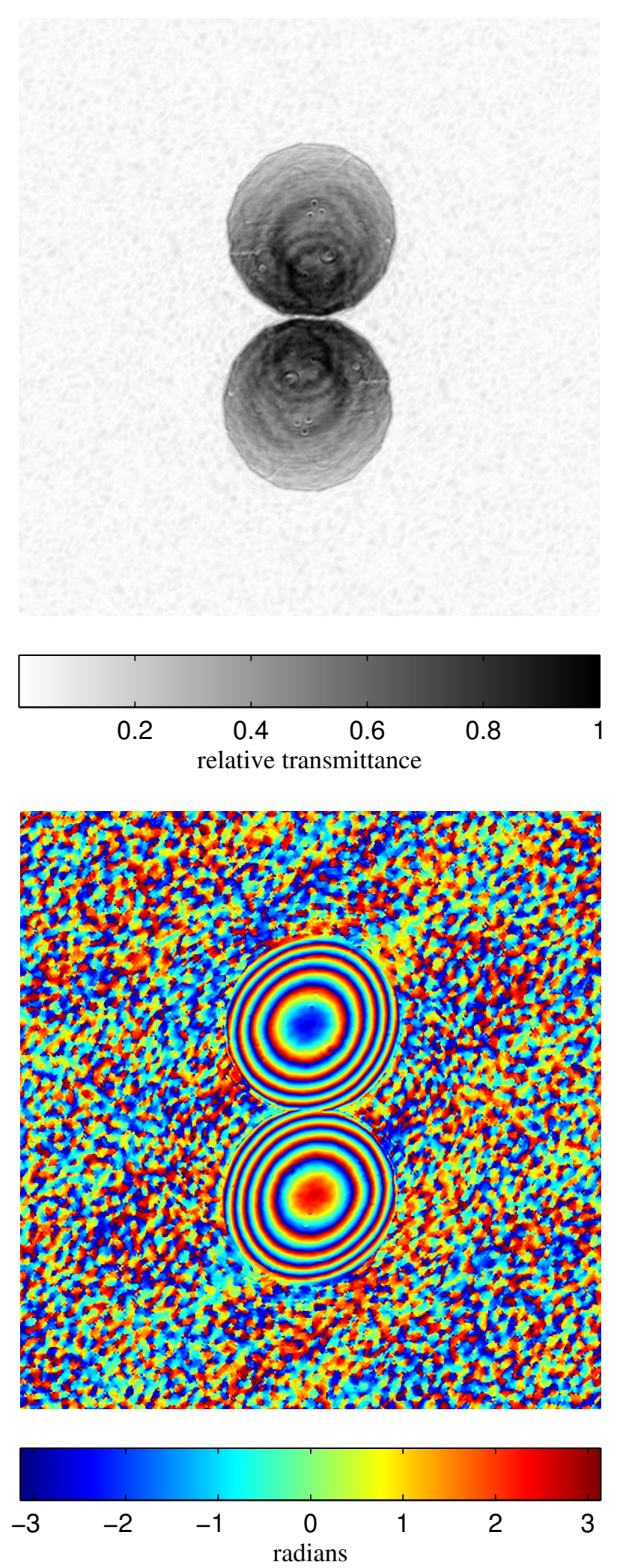

Fig. 7. One dOTF used for the SLM calibration. The arrays are $640 \times 640$ pixels wide. Upper: modulus. Lower: phase. the simulation: the pupil intensity is highest close to the diversity location, decreasing radially from that point.

The width of the pupil, compared to the dOTF array, is easy to determine from the data in Fig. 7. The intensity increases from the noise level to the mean pupil intensity $(\sim 0.5$ of the maximum $)$ in $\sim 4$ pixels. The pupils shown in Fig. 7 have a width of $186 \pm 1$ pixel, and therefore the sampling, as defined by Eq. (10), can be determined with a resolution of $\sim 1 \%$ to be 3.43 .

Fig. 7 also shows the phase in the pupil plane. Since we applied a large defocus, the most dominant feature is the concentric rings, caused by phase wrapping. We found that the noise levels were sufficiently small such that the original wavefronts were extremely easy to reconstruct by unwrapping the phase. To unwrap, we used the standard qualityguided algorithm, discussed for instance in [12].

\section{B. Determining SLM phase and amplitude re- sponse}

To measure the SLM response to a voltage change, we kept half of the SLM pixels at zero, and the control signal of the other pixels was set to a constant value. The dOTF method was used to determine the change of the pupil plane complex amplitudes in the region where the SLM was nonzero.

We noticed that the drift of the SLM created a significant change at lower spatial frequencies, especially tip/tilt, at time scales of $\sim 5-10$ minutes. Therefore, we had to record a new reference measurement (with all SLM pixels set to zero) separately for all the SLM modifications we wanted to determine.

Figure 8 shows the measured data and dOTF reconstructions for two cases: the reference and a case where the SLM control voltage is maximum in the lower part of the pupil. The area where the SLM pixels have been modified is clearly visible in the dOTF arrays. The dOTF modulus has a clear edge with a reduced intensity along the modification, exactly as in the simulations in Section 2.B.

Fig. 9 shows the arrays we used to determine the SLM change. As in Section 2.B, we subtracted the unwrapped dOTF phases to calculate the SLM phase change, and we took the ratio of the dOTF moduli to calculate the SLM transmittance change. To reduce the impact of noise, we applied median filtering (window size 5) to the unwrapped phase and the dOTF moduli. We also masked out $2 \%$ of the pupil at the edges to avoid dealing with the border effects. The masking was done only to compare 

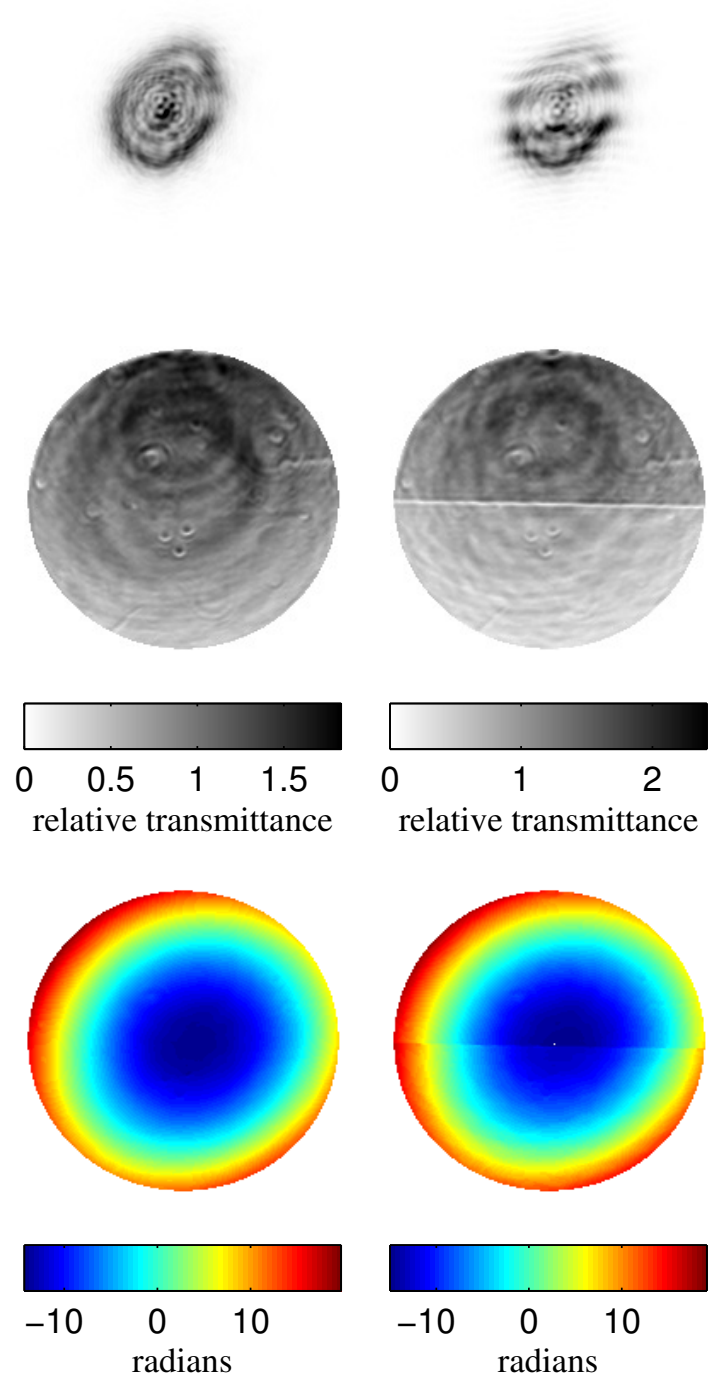

Fig. 8. Examples of SLM calibration. Upper row: recorded images. Middle row: dOTF moduli. Lower row: unwrapped dOTF phase. Left column: reference (all SLM pixels at zero). Right column: half of SLM pixels set to maximum control voltage.

the average values of the different half-pupils - it has no impact on the other aspects of the dOTF method.

Figure 9 shows an obvious change in the lower half of the pupil. Compared to the simulated case in Fig. 4, additional noise-like structure is present: the rms error of the upper and lower semi-pupils are $0.09 \mathrm{rad}$ and $0.16 \mathrm{rad}$, respectively, while the average difference between the semi-pupils is $1.6 \mathrm{rad}$. For the transmittance change, the values are 0.09 and 0.16 , and the difference between the semi-
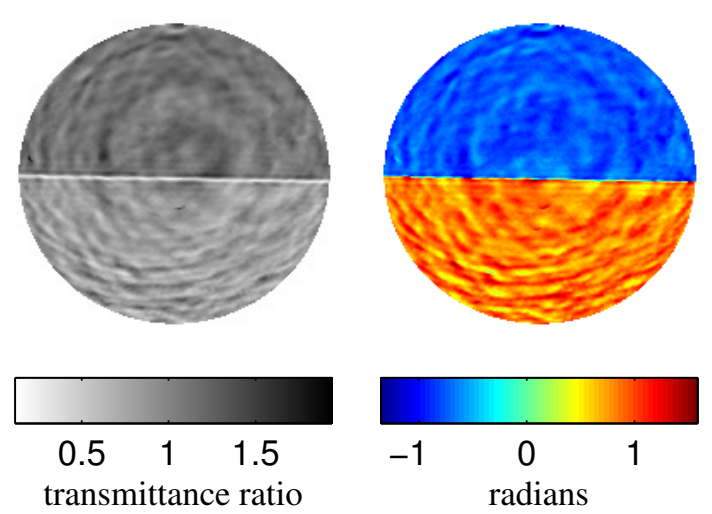

Fig. 9. Examples of SLM calibration. Left: ratio of dOTF moduli in Fig. 8, Right: difference of dOTF phase in Fig. 8 .

pupils is 0.25 .

It is a reasonable approximation that all the SLM pixels have an identical response and that the pixels are evenly distributed across the screen. Thus, the additional structure is caused by measurement errors. The drift of the SLM, together with a large defocus, can create the ring-like structures, and internal turbulence along the optical path is probably the reason for the speckle-like structure of the errors. Also Fresnel propagation effects from the SLM to the last pupil plane could have an impact, which would appear as additional border effects around the edge of the SLM modification.

Nevertheless, a good estimate of the average change can be obtained by taking an average over the whole area where a pupil modification is observed.

We repeated the measurements shown in Figs. 8 and 9 for several SLM control levels. Two independent series were recorded with different dOTF diversity locations. The resulting transmittance and phase difference are shown in Fig. 10. The independent measurement series are in excellent agreement: the difference of the two measurements is typically $\sim 1 \%$ of the average measurement. The transmittance is more difficult to measure with higher accuracy, when a large SLM control signal difference is applied; the difference grows to $\sim 3-5 \%$ at signals larger than 200. For the phase, the difference between two independent measurements is always less than $0.005 \mathrm{rad}$ (excluding the control signal 233, where it is $0.01 \mathrm{rad}$ ). This measured SLM response is in excellent agreement with the results reported in [11.

The maximum observed stroke was $1.6 \mathrm{rad}$, but 


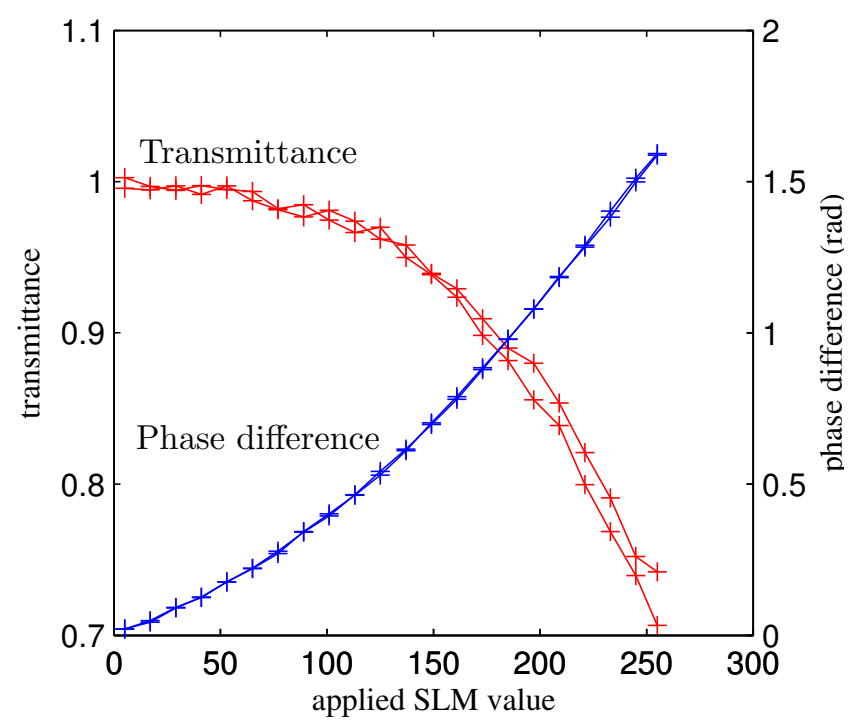

Fig. 10. SLM calibration results. Phase and transmittance as a function of applied SLM control signal value. Two independent measurement series are shown. Note that the phase plots overlap.

it comes at the cost of $\sim 25 \%$ loss in transmittance. If the maximum transmittance loss is restricted to $15 \%$, the available stroke is $\sim 1.2 \mathrm{rad}$.

\section{C. Determining SLM registration}

To determine the SLM registration with respect to the physical pupil, we first re-optimized the camera focus and the neutral density filters such that the same setting could be used later with our focal-plane wavefront-sensing algorithm. With these changes, the shortest possible exposure time $(80 \mu \mathrm{s})$ results in images at the optimal focus having a peak intensity $\sim 50 \%$ of the saturation level.

We applied the dOTF method with 6 different exposure times $(2-29 \mathrm{~ms})$ with a defocus of $\sim 17 \mathrm{rad}$ peak-to-valley, which was created by the DM. The resulting dynamic range of the recorded images was similar to what we reported in the previous section, although with larger errors caused by the longer integration times and smaller defocus giving less light on the peripheral camera pixels. However, we found the impact of these additional errors to be negligible for our purposes. Yet, a significant change, caused by the refocusing, was that the PSF sampling changed by $\sim 5 \%$ to a value of $w=3.26$ due to the lack of telecentricity of the beam reaching the camera.

In the same way as in the previous section, we introduced a change in phase with selected SLM pixels. We used the maximum control signal on stripe 66 pixels wide. We made 16 dOTF recordings using both vertical and horizontal stripes, which covered the pupil at equal intervals.

Then, we determined the location of the modified stripe in the pupil plane. We found it easiest to do this by using a gradient detection. The $\mathrm{x}$ and y-gradients of the dOTF phase were computed by subtracting two arrays, both shifted one pixel in opposite directions. Then, we took a squared sum of the $\mathrm{x}$ - and $\mathrm{y}$-gradients and filtered out $98 \%$ of the lowest values, thus keeping only the pixels describing the borders of the stripe. The pixels were used to compute two linear fits of the two edges of the stripe. Finally, a mean of these two lines was calculated, and it accurately represents the middle of the stripe. An example of this is shown in Fig. 11.

The fitting produces two sets of intersections two grids of 16 points. One of the sets is in the SLM pixel coordinates, the other in the physical pupil coordinates (whose sampling is determined by the size of the dOTF array).

Then, we calculate the optimal affine transform $\left(A_{t}\right)$ minimizing the error

$$
e=\sum_{i=1}^{16}\left\|A_{t}\left[\begin{array}{c}
x_{\text {meas }}(i) \\
y_{\text {meas }}(i) \\
1
\end{array}\right]-\left[\begin{array}{c}
x_{\mathrm{SLM}}(i) \\
y_{\mathrm{SLM}}(i)
\end{array}\right]\right\|^{2},
$$

where $\left(x_{\text {meas }}(i), y_{\text {meas }}(i)\right)$ are the coordinates determined from the dOTF reconstructions, $\left(x_{\mathrm{SLM}}(i), y_{\mathrm{SLM}}(i)\right)$ are the SLM pixels corresponding to the intersections determined in the pupil coordinates, $i$ refers to the intersections of the fitted lines, and $A_{t}$ is the transform expressed by a $2 \times 3$ matrix.

We used dOTF arrays having a size of $640 \times$ 640 pixels, and therefore the pupil coordinates $\left(x_{\text {meas }}(i), y_{\text {meas }}(i)\right)$ are within an array of $196 \times$ 196 pixels. That area maps to the SLM pixels having a size of $334 \times 334$ pixels and located within the device with $800 \times 600$ pixels. Thus, the mapping performs the interpolation that defines how many degrees of freedom we actually use to control the SLM.

We found that the intersections we determined in pupil coordinates can be mapped to SLM pixel coordinates with sub-pixel accuracy: the maximum error was 0.6 pixels, and the rms error was 0.4 pixels. The optimal transform was

$$
A_{t}=\left[\begin{array}{rrr}
1.687 & -0.029 & 235.9 \\
-0.044 & -1.704 & 483.1
\end{array}\right],
$$

which shows that a pure rotation, translation and 

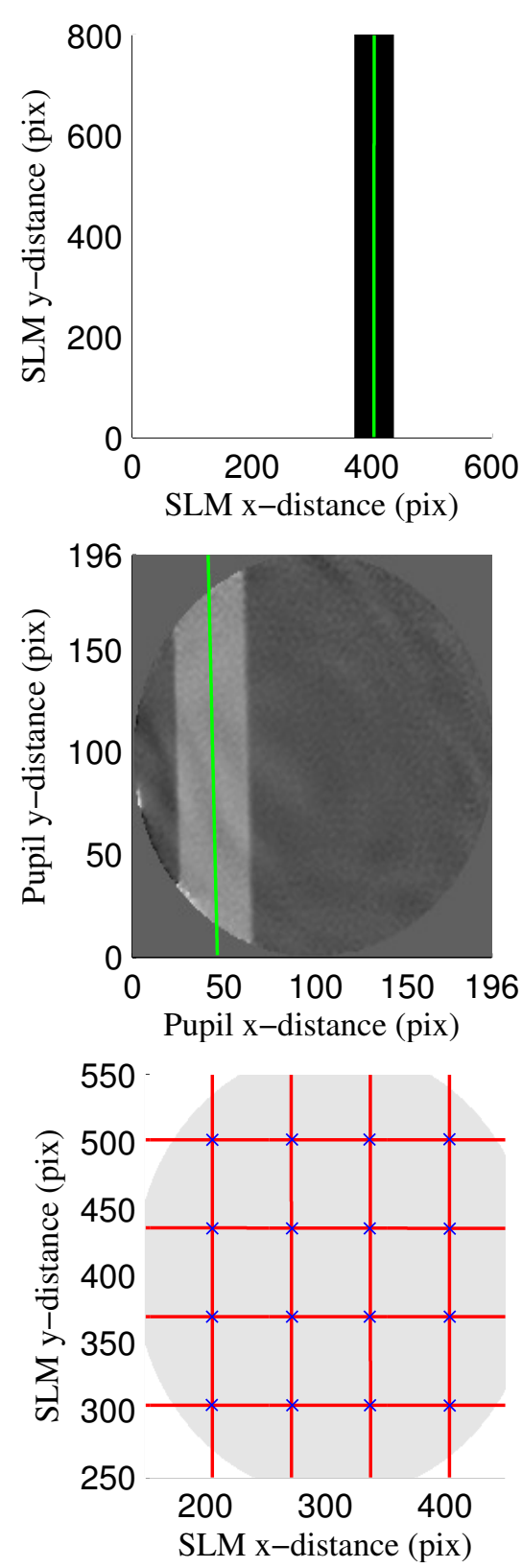

Fig. 11. An example of the SLM registration determination. Top: SLM pixels that were modified and a linear fit along its center. Middle: pupil phase recorded by the dOTF method and a linear fit. Bottom: red lines show all the SLM pixel modifications. Crosses show the intersections of dOTF measured lines mapped onto the SLM coordinates using an optimal affine transform. Gray area shows estimated pupil location.

scaling would be sub-optimal to describe the SLM position with respect to the pupil plane: the difference to the optimal translation would be about 1-2 SLM pixels at the peripheral pupil points. This is probably caused by a small tilt of the SLM or camera, compared to the optical axis. The values show that the pixel grids of the SLM and camera are rotated approximately $1-1.5^{\circ}$ with respect to each other, and this is also visible in Figs. 8, 9 and 11.

After having determined $A_{t}$ and the SLM response (as in Fig. 10), it is possible to employ standard image processing techniques to calculate the SLM control signal to create a desired phase change in the physical pupil.

\section{D. Performance with a focal plane sensing al- gorithm}

Finally, we tested how a phase-diversity based focal-plane wavefront sensing algorithm worked with a wavefront corrector calibrated as described before; PSF sampling determined as discussed in Section 2.C, SLM phase response and alignment determined as shown in Sections 4.B and 4.C, respectively. We use the Fast \& Furious algorithm due to its easy implementation. Our earlier work [2] has illustrated how it works with low-order modes correction, and here we show the results with a wavefront corrector having a spatial resolution of $196 \times 196$ control elements.

Fig. 12 illustrates the performance of F\&F. The left image was obtained when the camera was well focused, and low-order modes were corrected by the deformable mirror, but all the SLM pixels were set to zero. The right image shows the situation after the F\&F algorithm has converged. These two images - and all the images used by the algorithm are HDR compilations of 15 images with different exposure times (0.08-64 ms). The improvement in the image quality is obvious: the Strehl ratio (measured from the maximums of normalized images) increased from 0.80 to 0.93 .

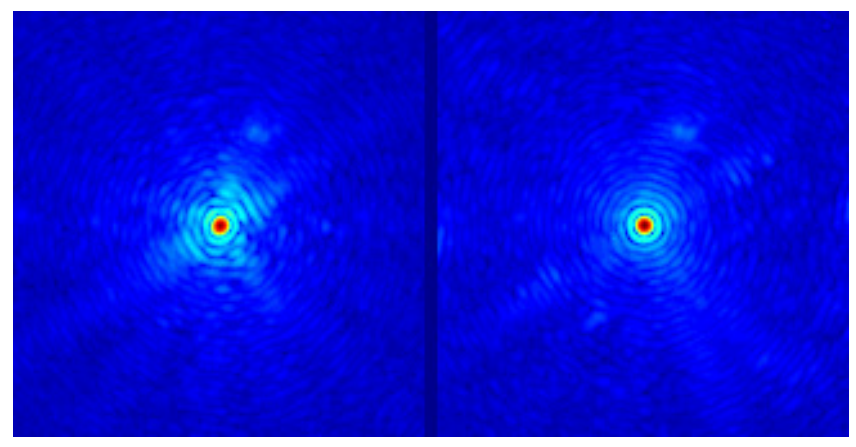

Fig. 12. Illustration of the Fast \& Furious performance: PSF images raised to the 0.2 power. The images have a size of $160 \times 160$ pixels. Left: before SLM correction (Strehl ratio 0.80). Right: after F\&F has converged (Strehl ratio 0.93). 
The remaining errors are likely caused by a ghost (internal reflection from SLM), saturation of the SLM (we limited the stroke to $1.2 \mathrm{rad}$ causing $16-$ $20 \%$ of the pixels to be saturated), and amplitude errors (caused by both the SLM, imperfectly modeled pupil and Fresnel propagation effects). However, a more detailed analysis is outside the scope of this paper, and we will address the issues in more detail in an upcoming publication.

\section{Conclusions}

We have shown that an extremely straightforward method - the differential OTF - can be used to calibrate a phase-diversity based focal-plane wavefront sensing algorithm that corrects the wavefront at a resolution of $\sim 200 \times 200$.

The dOTF method relies on localized diversities at the pupil border, which is a unique feature compared to other focal-plane wavefront sensing techniques. It requires no complicated hardware, physical movement of optical components or demanding numerical computations, and it therefore is an excellent option when an easily implementable way to accurately determine the pupil-plane electric field with a focal-plane camera is needed.

We have outlined how to theoretically predict the performance of the method, and our simulations are able to explain the experimental results - apart from the instability issues related to our hardware.

Based on theorical reasoning, we know that the ultimate resolution of the dOTF method is limited by the size and shape of the used diversity; it determines the convolution kernel that blurs the original complex amplitudes. In our experiments, the used diversity gives a maximum resolution between $\sim 50 \times 50$ and $\sim 150 \times 150$ pixels - but the resolving power can be different in vertical and horizontal directions. A visual inspection is in agreement with these values, although read-out noise and instabilities reduce the practical resolution of the instantaneous dOTF arrays.

To increase the calibration accuracy, we have used the fact that our wavefront corrector, an SLM, forms a fixed, rectangular array of evenly distributed pixels. We used the dOTF method to detect the sharp borders caused by modified wavefront blocks, and using those borders, we calculated the optimal affine transform projecting the SLM pixel locations onto the physical pupil plane. Based on the match of that mapping, we conclude that the locations of the SLM pixels are determined with an accuracy of $0.3 \%$ with respect to the pupil diameter.

This paper concentrates on concepts necessary for future extreme adaptive optics systems like the one necessary for the direct exoplanet imager ELT-PCS [13]. However, the methods discussed here are very versatile, and they can be put into use in many systems - in principle everywhere where measurements of optical aberrations are needed.

We have used the SLM to generate the localized diversities. However, they could as well be created by high-resolution deformable mirrors such as in [14]. Also, additional simple mechanics at the pupil edge could be used to introduce a small, localized obstruction as a diversity.

The technique is best suited for applications where a monochromatic light source is available. If the spectral bandwidth is increased, the diffraction features further off-axis are blurred, which implies that higher spatial frequency information in the dOTF reconstruction is lost. However, lower spatial frequencies can still be recovered to some extent [5]. High-contrast speckle-nulling experiments have demonstrated good success using $10 \%$ bandwidth [15, and probably a similar bandwidth is feasible also with the dOTF method.

Cases that will benefit from the dOTF calibration are test benches demonstrating techniques for ultra-high contrast imaging needed in space-based exo-planet detection (e.g., HCIT [16]), experiments for ground-based extreme adaptive optics (ExAO testbed [17, HOT [18, FFREE [19]) and the pathfinder XAO instruments (GPI [20], SPHERE [21]).

The main issue with the dOTF method is the measurement noise. We need a recording time of 2-4 min for each dOTF measurement to reduce the read-out noise and achieve the required high dynamic range. During this recording process, the system behavior should be stationary. However, we observed the drift of the SLM when trying to increase the measurement time to more than $5 \mathrm{~min}$; a real DM would likely be better. Internal turbulence in the optical setup can also be a serious problem.

Another point of concern is the resolution of the pupil plane measurement. It is ultimately limited by the size of the localized diversity, and we proposed to create it with the wavefront corrector elements. Thus, it is necessary that the elements are evenly spaced across the pupil and that they have identical response. What we can measure is limited to the response of larger blocks of the correction elements - when a typical deformable mirror is used to introduce the diversity, the coupling of the actuators is an additional challenge.

Our future work will concentrate on demonstrating how the dOTF method can be used in a wider 
range of optical experiments. These include additional optics such as apodizers and coronagraphs. In addition, we will include a more detailed analysis of the light propagation effects from the wavefront corrector to the last pupil plane.

\section{Acknowledgments}

We thank Gerard van Harten and Tim van Werkhoven for assistance with the spatial light modulator and optical experiments.

\section{References}

[1] C. U. Keller, V. Korkiakoski, N. Doelman, R. Fraanje, R. Andrei, and M. Verhaegen, "Extremely fast focal-plane wavefront sensing for extreme adaptive optics," (2012), vol. 8447 of Proc. SPIE, pp. 844721-844721.

[2] V. Korkiakoski, C. U. Keller, N. Doelman, R. Fraanje, R. Andrei, and M. Verhaegen, "Experimental validation of optimization concepts for focal-plane image processing with adaptive optics," (2012), vol. 8447 of Proc. SPIE, pp. 84475Z-84475Z.

[3] J. L. Codona, "Theory and application of differential otf (dotf) wavefront sensing," (2012), vol. 8447 of Proc. SPIE, pp. $84476 \mathrm{P}-84476 \mathrm{P}$.

[4] J. L. Codona and N. Doble, "Experimental evaluation of differential otf (dotf) wavefront sensing," (2012), vol. 8447 of Proc. SPIE, pp. 84476R84476R.

[5] J. L. Codona, "Differential optical transfer function wavefront sensing," Optical Engineering 52, pp. 097105-097105 (2013).

[6] R. W. Gerchberg and W. O. Saxton, "A practical algorithm for the determination of the phase from image and diffraction plane pictures," Optik 35, 237 (1972).

[7] J. R. Fienup, "Phase retrieval algorithms: a comparison," Applied Optics21, 2758-2769 (1982).

[8] T. P. Zielinski, "Robust Image-Based Wavefront Sensing," Ph.D. thesis, University of Rochester (2011).

[9] G. R. Brady and J. R. Fienup, "Measurement range of phase retrieval in optical surface and wavefront metrology," Appl. Opt.48, 442 (2009).

[10] S. Mann and R. Picard, "Being 'undigital' with digital cameras: Extending dynamic range by combining differently exposed pictures," Tech. Rep. 323, M.I.T. Media Lab Perceptual Computing Section, Boston, Massachusetts (1994).

[11] J. Reményi, P. Várhegyi, L. Ódomján, P. Koppa, and E. Lorincz, "Amplitude, Phase, and Hybrid Ternary Modulation Modes of a Twisted-Nematic Liquid-Crystal Display at 400 nm," Appl. Opt.42, 3428-3434 (2003).
[12] D. C. Ghiglia and M. D. Pritt, Two-Dimensional Phase Unwrapping-Theory, Algorithms, and Software (Wiley-Interscience, 1998).

[13] M. Kasper, D. Mawet, and C. Verinaud, "Roadmap for PCS," in "Third International Conference on Adaptive Optics for Extremely Large Telescopes," (2013).

[14] L. A. Poyneer, B. Bauman, S. Cornelissen, J. Isaacs, S. Jones, B. A. Macintosh, and D. W. Palmer, "The use of a high-order mems deformable mirror in the gemini planet imager," in "SPIE MOEMS-MEMS," (2011), Proc. SPIE, pp. 793104-793104.

[15] A. Give'on, B. Kern, S. Shaklan, D. C. Moody, and L. Pueyo, "Broadband wavefront correction algorithm for high-contrast imaging systems," (2007), vol. 6691 of Proc. SPIE, pp. 66910A-66910A-11.

[16] A. E. Lowman, J. T. Trauger, B. Gordon, J. J. Green, D. Moody, A. F. Niessner, and F. Shi, "High-contrast imaging testbed for the Terrestrial Planet Finder coronagraph," (2004), vol. 5487 of Proc. SPIE, pp. 1246-1254.

[17] S. A. Severson, B. Bauman, D. Dillon, J. Evans, D. Gavel, B. Macintosh, K. Morzinski, D. Palmer, and L. Poyneer, "The extreme adaptive optics testbed at UCSC: current results and coronagraphic upgrade," (2006), vol. 6272 of Proc. SPIE, pp. 62722J-62722J.

[18] E. Vernet, M. Kasper, C. Vérinaud, E. Fedrigo, S. Tordo, N. Hubin, S. Esposito, E. Pinna, A. Puglisi, A. Tozzi, A. G. Basden, S. J. Goodsell, G. D. Love, and R. M. Myers, "Extreme adaptive optics system optimization with the high order test bench," (2006), vol. 6272 of Proc. SPIE, p. 62722K.

[19] J. Antichi, C. Vérinaud, O. Preis, A. Delboulbé, G. Zins, P. Rabou, J.-L. Beuzit, S. Dandy, J.-F. Sauvage, T. Fusco, E. Aller-Carpentier, M. Kasper, and N. Hubin, "FFREE: a Fresnel-FREE demonstrator for the common-path optics within EPICS," in "In the Spirit of Lyot 2010," (2010).

[20] B. Macintosh, J. Graham, D. Palmer, R. Doyon, D. Gavel, J. Larkin, B. Oppenheimer, L. Saddlemyer, J. K. Wallace, B. Bauman, J. Evans, D. Erikson, K. Morzinski, D. Phillion, L. Poyneer, A. Sivaramakrishnan, R. Soummer, S. Thibault, and J.-P. Veran, "The Gemini Planet Imager," (2006), vol. 6272 of Proc. SPIE, p. 62720L.

[21] T. Fusco, G. Rousset, J.-F. Sauvage, C. Petit, J.L. Beuzit, K. Dohlen, D. Mouillet, J. Charton, M. Nicolle, M. Kasper, P. Baudoz, and P. Puget, "High-order adaptive optics requirements for direct detection of extrasolar planets: Application to the SPHERE instrument," Opt. Express 14, 7515-7534 (2006). 